\title{
Perfil epidemiológico de intoxicação exógena por medicamentos em Brasília
}

\author{
Epidemiological Profile of Exogenous Drug Intoxication in Brasília
}

\author{
Jéssica Yohanna Silva Soares ${ }^{1}$ \\ Orcid: https://orcid.org/0000-0002-4056-317X \\ Igor Araújo Verri ${ }^{3}$ \\ Orcid: https://orcid.org/0000-0001-9835-9879
}

\author{
Beatriz Matos de Lima ${ }^{2}$ \\ Orcid: https://orcid.org/0000-0003-2446-2712 \\ Stefan Vilges de Oliveira ${ }^{4}$ \\ Orcid: https://orcid.org/0000-0002-5493-2765
}

\begin{abstract}
Resumo
Introdução: No Brasil, as intoxicações exógenas são problemas de saúde pública extremamente relevantes, uma vez que apresentam elevada frequência e morbidade na população geral do país. Objetivo: Descrever medidas de intervenção eficazes para redução de casos e óbitos por intoxicações exógenas por medicamentos baseadas no perfil epidemiológico dessas intoxicações na cidade de Brasília-DF. Método: estudo epidemiológico, descritivo, em que foram analisados dados do Sistema de Informação de Agravos de Notificação, referentes à cidade de Brasília-DF compreendendo os casos notificados de intoxicação exógena por medicamentos no período entre os anos de 2011 a 2017. Para subsidiar a discussão e a proposta de intervenção, foi realizada uma revisão integrativa da literatura nas plataformas Scielo e LILACS. Resultados: 22 publicações foram selecionadas após aplicação dos critérios de análise e serviram como base para a proposta de intervenção do presente artigo. Conclusão: Nota-se a importância de estratégias como a promoção de qualidade de vida, educação, proteção, recuperação para a prevenção do suicídio e implantação de embalagens de medicamentos de proteção à criança dentre outras estratégias de intervenções.
\end{abstract}

Palavras-chave: vigilância epidemiológica; medicamentos; toxicologia; intoxicação; projetos

\begin{abstract}
Introduction: In Brazil, exogenous intoxications are extremely relevant public health problems, since they have a high frequency and morbidity in the general population of the country. Aim: To describe effective intervention measures to reduce cases and deaths from drug exogenous intoxication based on the epidemiological profile of these intoxications in the city of Brasilia-DF. Method: an epidemiological, descriptive study, where data from the Information System for Notifiable Diseases were analyzed, referring to the city of Brasilia-DF, including the reported cases of exogenous drug intoxication in the period from 2011 to 2017, were analyzed. To support the discussion and the intervention proposal, a literature review was carried out on Scielo and LILACS databases. Results: 22 publications were selected after applying the analysis criteria and served as the basis for the intervention proposal of this article. Conclusion: We note the importance of strategies such as the promotion of quality of life, education, protection, recovery for the prevention of suicide, implementation of child protection medication packaging, among other intervention strategies.
\end{abstract}

Keywords: epidemiological monitoring; pharmaceutical preparations; toxicology; poisoning. Projects

\footnotetext{
${ }^{1}$ Universidade Federal de Uberlândia, MG, Brasil. E-mail: jyohannass@gmail.com

${ }^{2}$ Universidade Federal de Uberlândia. MG, Brasil. E-mail: bbeatriz.matos@gmail.com

${ }^{3}$ Universidade Federal de Uberlândia. MG, Brasil. E-mail: igorverri@outlook.com

${ }^{4}$ Universidade Federal de Uberlândia. MG, Brasil. E-mail: igorverri@outlook.com
} 


\section{Introdução}

No Brasil, as intoxicações exógenas são problemas de saúde pública extremamente relevantes, uma vez que apresentam elevada frequência e morbidade na população geral do país ${ }^{1}$. Conforme a base do Sistema Nacional de Informações Tóxico Farmacológicas do ano de 2017, observou- se que $27,11 \%$ das intoxicações registradas no país foram por medicamentos, contra $3,34 \%$ por agrotóxico agrícola e 1,09\% por agrotóxico doméstico. No que se refere aos óbitos causados por intoxicação humana, notou-se que os índices mais elevados foram: $30,42 \%$ por agrotóxicos de uso agrícola; 25 $\%$ por medicamentos; $8 \%$ por drogas de abuso e $8 \%$ por produtos químicos industriais. Logo, no Brasil, os medicamentos são causas relevantes de intoxicação e de óbito por agentes danosos ${ }^{2}$.

A indústria farmacêutica tem se desenvolvido nas últimas décadas gerando novos produtos e exercendo importantes transformações no âmbito dos medicamentos em esfera mundial. Devido à grande diversidade de fármacos à disposição no comércio brasileiro, surgem também problemas como a intoxicação por esses produtos ${ }^{3}$. Nota-se, assim, que tal dilema é uma questão de saúde pública, a qual necessita da devida atenção, tanto pelos profissionais dessa área e pelos órgãos públicos, quanto pela sociedade como um todo ${ }^{4,5}$. Além disso, tal fenômeno pode ser evitado, sobretudo, pela identificação prévia e medidas que visem mitigar possíveis dados 5 .

Segundo dados do Ministério da Saúde do Brasil, o Distrito Federal apresenta elevada frequência anual de intoxicações exógenas, quando comparadas com outras capitais brasileiras ${ }^{4}$. Brasília, quando comparada com outras capitais de mesmo porte, no mesmo período, apresenta prevalência média anual de intoxicações por medicamentos maior que Salvador e Fortaleza. No entanto, em relação às unidades federativas, o Distrito Federal é o $10^{\circ}$ com maior incidência de intoxicações por medicamentos, uma posição razoavelmente expressiva ${ }^{6}$.

Diante do exposto, este estudo tem por objetivo descrever medidas de intervenção eficazes para redução de casos e óbitos por intoxicações exógenas por medicamentos baseadas no perfil epidemiológico dessas intoxicações na cidade de Brasília - DF no período de 20112017.

\section{Materiais e Métodos}

\section{Amostra e tipo de estudo}

Trata-se de um estudo ecológico exploratório com revisão da literatura, elaborado a partir dos dados disponibilizados pelo Departamento de Informática para o Sistema Único de Saúde (DATASUS) e provenientes do Sistema de Informação de Agravos de Notificação (SINAN), de responsabilidade da Secretaria de Vigilância em Saúde. Esse sistema armazena informações de notificação e investigação de doenças e agravos que constam na Lista Nacional de Notificação Compulsória, regida pela Portaria de Consolidação $n^{\circ} 4{ }^{4}$.

O foco do estudo foi a capital federal, Brasília, que possuia população estimada de 2.974.703 habitantes, em 2018. Quanto à assistência à saúde, a cidade contava com 1756 estabelecimentos de saúde e 5294 leitos de internação em 2009. Quanto à economia, o rendimento mensal per capita em 2016 foi o sétimo maior do Brasil, contudo $30,9 \%$ da população recebia menos de $1 / 2$ salário mínimo por mês, o que demarca a desigualdade social da capital ${ }^{7}$.

\section{Delineamento da pesquisa}

As informações foram obtidas e filtradas por meio do sistema TabNet, que é o tabulador de dados do DATASUS. Nesse sistema, selecionou-se a seção 
"Epidemiológicas e Morbidade" e o grupo "Doenças e Agravos de Notificação". Dentre os agravos, foi escolhida a opção "Intoxicações Exógenas" cuja região de notificação foi o Distrito Federal, na cidade de Brasília.

Posteriormente, as intoxicações foram filtradas para o agente tóxico "medicamento". A série temporal analisada inicia-se em 2011, pois a quantidade de notificações anteriores a esse ano é ínfima, provavelmente devido à subnotificação; e finaliza-se em 2017, último ano com dados disponíveis.

\section{Critérios de Inclusão e Exclusão}

Para subsidiar a discussão e a proposta de intervenção para redução das intoxicações exógenas por medicamentos em Brasília, foi realizada revisão integrativa da literatura, com auxílio da ferramenta PRISMA ${ }^{\circledR}$ (Figura 4). As bases de dados pesquisadas foram Scielo e LILACS, que estão entre as principais da América Latina.
Na fase de identificação utilizaramse os termos "intoxicação" e "medicamentos", ambos escolhidos dentre os Descritores em Saúde (DeCS) da Biblioteca Virtual em Saúde (BVS). Em ambas as bases de dados foi utilizada a combinação de busca "intoxicação AND medicamentos". A partir dos resultados obtidos, foram incluídos todos os artigos publicados de 2012 a 2017, nos idiomas português e inglês. Após aplicar os critérios de inclusão, foram excluídas publicações duplicadas entre as bases de dados.

$\mathrm{Na}$ fase de análise, o resumo dos artigos foi lido de forma independente entre os autores que aplicaram os seguintes critérios de exclusão: não abordar intoxicações exógenas por medicamento; não trazer uma proposta de intervenção. Por fim, sucedeu-se a leitura completa dos artigos incluídos e foi confeccionada uma tabela com as informações: título, autores, ano de publicação, objetivo geral, principais resultados e proposta de intervenção (Quadro 1).

Quadro 1: Resultados da revisão bibliográfica

\begin{tabular}{|c|c|c|c|c|}
\hline Autores & Ano & Objetivo Geral & Principais Resultados & Proposta de Intervenção \\
\hline $\begin{array}{l}\text { Magalhães } \\
\text { et al. }{ }^{1}\end{array}$ & 2014 & $\begin{array}{l}\text { Investigar as características } \\
\text { das vítimas de tentativa de } \\
\text { suicídio atendidas em } \\
\text { serviço pré-hospitalar e o } \\
\text { tempo consumido nessa } \\
\text { fase do atendimento. }\end{array}$ & $\begin{array}{c}\text { As mulheres, com idade } \\
\text { superior à dos homens, foram } \\
\text { as que mais tentaram } \\
\text { suicídio, e a intoxicação por } \\
\text { medicamentos foi o método } \\
\text { mais utilizado. O tempo } \\
\text { gasto para o atendimento pré- } \\
\text { hospitalar variou entre } 34,4 \text { e } \\
40,5 \text { minutos. }\end{array}$ & $\begin{array}{l}\text { Estabelecer um protocolo de } \\
\text { intervenções e um rigoroso } \\
\text { controle na distribuição de } \\
\text { medicamentos e agrotóxicos. } \\
\text { Capacitar e articular serviços de } \\
\text { saúde para lidar com o } \\
\text { comportamento suicida, } \\
\text { garatindo abordagem adequada e } \\
\text { seguimento do tratamento. }\end{array}$ \\
\hline $\begin{array}{c}\text { Mota DM et } \\
a l . .^{5}\end{array}$ & 2012 & $\begin{array}{c}\text { Descrever o perfil } \\
\text { epidemiológico da } \\
\text { mortalidade por intoxicação } \\
\text { com medicamentos na } \\
\text { população do Brasil entre } \\
1996 \text { e } 2005 .\end{array}$ & $\begin{array}{l}\text { A maioria dos óbitos ocorreu } \\
\text { no sexo masculino, em } \\
\text { solteiros, na faixa etária de } \\
\text { 20-39 anos. A principal causa } \\
\text { foi autointoxicação } \\
\text { intencional. A taxa de } \\
\text { mortalidade foi maior no } \\
\text { Centro-Oeste e os Anos } \\
\text { Potenciais de Vida Perdidos } \\
\text { aumentou } 15,5 \% \text { no período. }\end{array}$ & $\begin{array}{l}\text { Fica evidente a importância do } \\
\text { uso potencial do Sistema de } \\
\text { Informações sobre Mortalidade } \\
\text { como fonte de informação em } \\
\text { farmacoepidemiologia para } \\
\text { subsidiar o aprimoramento de } \\
\text { políticas de vigilância sanitária. }\end{array}$ \\
\hline $\begin{array}{l}\text { Amorim } \\
\text { MLP et al. }\end{array}$ & 2017 & $\begin{array}{l}\text { Analisar o perfil clínico e } \\
\text { epidemiológico do }\end{array}$ & $\begin{array}{l}92,2 \% \text { dos envenenamentos } \\
\text { foram acidentais; } 56,3 \%\end{array}$ & $\begin{array}{l}\text { Ações de vigilância e } \\
\text { assistência; mobilização da }\end{array}$ \\
\hline
\end{tabular}




\begin{tabular}{|c|c|c|c|c|}
\hline & & $\begin{array}{l}\text { envenenamento em } \\
\text { crianças e adolescentes com } \\
\text { menos de } 12 \text { anos de idade. }\end{array}$ & $\begin{array}{l}\text { foram por substâncias } \\
\text { químicas; e } 43,7 \% \text { por } \\
\text { animais peçonhentos. A } \\
\text { frequência de intoxicação } \\
\text { química foi maior em } \\
\text { crianças menores de } 5 \text { anos, } \\
\text { não houve diferença entre os } \\
\text { sexos e } 99,6 \% \text { delas } \\
\text { ocorreram em casa. }\end{array}$ & $\begin{array}{l}\text { sociedade para desenvolver } \\
\text { campanhas junto a profissionais } \\
\text { de saúde, educadores e } \\
\text { população; formação de } \\
\text { profissionais que atuem de forma } \\
\text { ativa na prevenção, diagnóstico e } \\
\text { tratamento desses agravos; } \\
\text { pressionar a aprovação dos } \\
\text { projetos de lei ligados à } \\
\text { prevenção de acidentes com } \\
\text { crianças que tramitam no } \\
\text { Congresso Nacional. }\end{array}$ \\
\hline $\begin{array}{c}\text { Teles ADS } \\
\text { et al. }{ }^{10}\end{array}$ & 2013 & $\begin{array}{c}\text { Descrever o perfil e a } \\
\text { evolução das intoxicações } \\
\text { em Feira de Santana, Bahia, } \\
\text { de } 2007 \text { a } 2010 \text {, e analisar a } \\
\text { morbimortalidade causada } \\
\text { por medicamentos. }\end{array}$ & $\begin{array}{l}\text { 33\% das intoxicações foram } \\
\text { por medicação e a tentativa } \\
\text { de suicídio foi a principal } \\
\text { causa. A maioria dos casos } \\
\text { ocorreu no sexo feminino, } \\
\text { em crianças de } 1 \text { a } 4 \text { anos e } \\
\text { em adultos jovens. A } \\
\text { letalidade em Feira de } \\
\text { Santana foi maior que a } \\
\text { média regional e nacional. }\end{array}$ & $\begin{array}{l}\text { A Vigilância em Saúde deve } \\
\text { priorizar ações educativas, } \\
\text { preventivas e intersetoriais, que } \\
\text { assegurem o uso correto de } \\
\text { medicamentos, bem como } \\
\text { investir na sensibilização } \\
\text { continuada de profissionais da } \\
\text { Atenção Básica para melhorar a } \\
\text { notificação deste agravo. }\end{array}$ \\
\hline $\begin{array}{c}\text { Vieira et } \\
\text { al. }{ }^{11}\end{array}$ & 2015 & $\begin{array}{l}\text { Apresentar o perfil das } \\
\text { vítimas de tentativas de } \\
\text { suicídio por intoxicações } \\
\text { exógenas ocorridas no } \\
\text { município de Barra do } \\
\text { Garças. }\end{array}$ & $\begin{array}{l}\text { Os agentes tóxicos de maior } \\
\text { frequência foram } \\
\text { medicamentos }(50 \%) \text { e } \\
\text { agrotóxicos }(26,2 \%) \text {. As } \\
\text { tentativas foram mais } \\
\text { frequentes entre o gênero } \\
\text { feminino }(71,1 \%) \text { e em } \\
\text { adultos. }\end{array}$ & $\begin{array}{l}\text { Campanhas de conscientização } \\
\text { sobre o uso racional de } \\
\text { medicamentos, maior controle } \\
\text { sobre a venda de agrotóxicos e } \\
\text { realização de programas de } \\
\text { apoio e atendimento às vítimas. }\end{array}$ \\
\hline $\begin{array}{l}\text { Domingos } \\
\text { SM et al. }{ }^{12}\end{array}$ & 2016 & $\begin{array}{c}\text { Descrever o perfil das } \\
\text { intoxicações que levaram à } \\
\text { internação de crianças no } \\
\text { Hospital Universitário } \\
\text { Regional de Maringá de } \\
2006 \text { a } 2011 .\end{array}$ & $\begin{array}{c}\text { Medicamentos foram os } \\
\text { principais responsáveis pelas } \\
\text { intoxicações que causaram as } \\
\text { internações, a maioria das } \\
\text { internações ocorreu na faixa } \\
\text { etária de } 1 \text { a } 4 \text { anos e no sexo } \\
\text { masculino. }\end{array}$ & $\begin{array}{l}\text { Capacitar profissionais da saúde } \\
\text { em todos os níveis, atendimento } \\
\text { emergencial melhorado, leis de } \\
\text { promoção e incentivo à produção } \\
\text { de embalagens de proteção à } \\
\text { criança, campanhas de precaução } \\
\text { a acidentes infantis. }\end{array}$ \\
\hline $\begin{array}{l}\text { Antunes F. } \\
\text { Oliveira } \\
\text { MLFD }^{13}\end{array}$ & 2013 & $\begin{array}{l}\text { Caracterizar o perfil clínico } \\
\text { e demográfico dos } \\
\text { pacientes hospitalizados } \\
\text { por intoxicação por } \\
\text { substâncias de abuso de um } \\
\text { hospital universitário, entre } \\
2004 \text { e } 2010 .\end{array}$ & $\begin{array}{c}\text { O álcool foi a substância } \\
\text { relacionada ao maior número } \\
\text { de internações }(77 \%) \text {, } \\
\text { seguida pelos medicamentos } \\
\text { psicoativos }(14 \%) \text {. Os } \\
\text { pacientes em sua maioria são } \\
\text { homens em idade } \\
\text { economicamente ativa, com } \\
\text { um número relevante de } \\
\text { mortes. }\end{array}$ & $\begin{array}{c}\text { O enfermeiro deve desenvolver } \\
\text { atividades preventivas, } \\
\text { monitoramento e orientação, } \\
\text { além de capacitar os } \\
\text { profissionais envolvidos, por } \\
\text { meio de educação continuada, } \\
\text { para realizar um cuidado mais } \\
\text { humano e menos estigmatizante } \\
\text { para os dependentes químicos. }\end{array}$ \\
\hline $\begin{array}{l}\text { Carvalho } \\
\text { ILDN et } \\
\text { al. }^{14}\end{array}$ & 2017 & $\begin{array}{c}\text { Caracterizar as intoxicações } \\
\text { por psicofármacos com } \\
\text { motivação suicida em } \\
\text { idosos. }\end{array}$ & $\begin{array}{l}\text { Intoxicações medicamentosas } \\
\text { com motivação suicida são } \\
\text { mais frequentes entre } 60 \text { a } 69 \\
\text { anos, no sexo feminino e em } \\
\text { idosos aposentados. }\end{array}$ & $\begin{array}{l}\text { Promoção do envelhecimento } \\
\text { ativo promovida por equipe } \\
\text { multiprofissional de saúde da } \\
\text { atenção básica, acesso e uso }\end{array}$ \\
\hline
\end{tabular}




\begin{tabular}{|c|c|c|c|c|}
\hline & & & & $\begin{array}{l}\text { racional de psicofármacos e } \\
\text { psicoterapia. }\end{array}$ \\
\hline $\begin{array}{l}\text { Moreira DL } \\
\text { et al. } .^{15}\end{array}$ & 2015 & $\begin{array}{l}\text { Caracterizar os pacientes } \\
\text { atendidos por tentativa de } \\
\text { suicídio, no Centro de } \\
\text { Assistência Toxicológica } \\
\text { de um hospital de } \\
\text { Fortaleza. }\end{array}$ & $\begin{array}{l}\text { Predominaram atos suicidas } \\
\text { por automedicação, no sexo } \\
\text { feminino, nos adultos jovens, } \\
\text { nos estudantes e nos } \\
\text { indivíduos provenientes de } \\
\text { zonas urbanas. }\end{array}$ & $\begin{array}{l}\text { Suporte psicológico ao paciente } \\
\text { com ideais suicidas; } \\
\text { planejamento de políticas } \\
\text { públicas efetivas, de promoção e } \\
\text { prevenção da saúde; esforço de } \\
\text { gestores, profissionais de saúde e } \\
\text { familiares em ações de } \\
\text { prevenção ao suicídio. }\end{array}$ \\
\hline $\begin{array}{l}\text { Oliveira } \\
\text { JDFMD }^{16}\end{array}$ & 2017 & $\begin{array}{c}\text { Descrever óbitos e } \\
\text { internações por intoxicação } \\
\text { com medicamentos no } \\
\text { estado e município de São } \\
\text { Paulo, e investigar os } \\
\text { fatores associados. }\end{array}$ & $\begin{array}{l}\text { Mulheres e idosos } \\
\text { apresentaram maiores } \\
\text { coeficientes de internação e } \\
\text { se observou tendência } \\
\text { crescente na mortalidade por } \\
\text { intoxicação medicamentosa. }\end{array}$ & $\begin{array}{l}\text { Estratégias de prevenção ao } \\
\text { suicídio, medidas de educação } \\
\text { em saúde. Incentivo ao uso } \\
\text { racional, controle de acesso e } \\
\text { acompanhamento de práticas } \\
\text { associadas a medicamentos e } \\
\text { capacitação de prescritores. }\end{array}$ \\
\hline $\begin{array}{l}\text { Carvalho } \\
\text { IV }^{17}\end{array}$ & 2013 & $\begin{array}{c}\text { Analisar a incidência de } \\
\text { eventos adversos aos } \\
\text { medicamentos e adesão à } \\
\text { farmacoterapia de uso } \\
\text { contínuo em pacientes } \\
\text { pediátricos em uma unidade } \\
\text { de emergência. }\end{array}$ & $\begin{array}{l}\text { Há alta incidência de eventos } \\
\text { adversos a medicamentos na } \\
\text { admissão de pacientes } \\
\text { pediátricos em unidade de } \\
\text { emergência, representando } \\
11,5 \% \text { do total, das quais } \\
39,3 \% \text { decorrentes do uso } \\
\text { inadequado. }\end{array}$ & $\begin{array}{l}\text { O desenvolvimento de ações de } \\
\text { farmacovigilância e programas } \\
\text { de educação social são essenciais } \\
\text { para a promoção do uso racional } \\
\text { de medicamentos, podendo } \\
\text { reduzir significativamente os } \\
\text { eventos adversos. }\end{array}$ \\
\hline $\begin{array}{l}\text { Oliveira } \\
\text { FFS, } \\
\text { Suchara } \\
\text { EA }^{18}\end{array}$ & 2014 & $\begin{array}{c}\text { Caracterizar o perfil } \\
\text { epidemiológico das } \\
\text { intoxicações exógenas } \\
\text { ocorridas em crianças e } \\
\text { adolescentes em Barra do } \\
\text { Garças. }\end{array}$ & $\begin{array}{c}\text { As intoxicações exógenas } \\
\text { ocorreram } \\
\text { predominantemente em } \\
\text { crianças até } 4 \text { anos por meio } \\
\text { do consumo de alimentos ou } \\
\text { bebidas e de forma acidental. }\end{array}$ & $\begin{array}{l}\text { Investimento em medidas de } \\
\text { controle sanitário, e de educação } \\
\text { para prevenir a ocorrência dessas } \\
\text { intoxicações. }\end{array}$ \\
\hline $\begin{array}{c}\text { Oliveira } \\
\text { JDFMD et } \\
\text { al. }{ }^{19}\end{array}$ & 2017 & $\begin{array}{l}\text { Apresentar a avaliação da } \\
\text { magnitude e da tendência } \\
\text { dos óbitos por intoxicação } \\
\text { medicamentosa no estado } \\
\text { de São Paulo, Brasil, no } \\
\text { período de } 1996 \text { a } 2012 .\end{array}$ & $\begin{array}{c}\text { Observou-se tendência } \\
\text { crescente de mortalidade } \\
\text { ajustada a partir de } 2005 \text {, que } \\
\text { se acentuou a partir de } 2009 \text {. } \\
\text { A autointoxicação } \\
\text { medicamentosa intencional } \\
\text { foi a que mais cresceu no } \\
\text { período, assim como a } \\
\text { mortalidade entre os mais } \\
\text { jovens. }\end{array}$ & $\begin{array}{l}\text { Ações educativas e reguladoras } \\
\text { para sensibilização contínua de } \\
\text { profissionais de saúde, para } \\
\text { assegurar a geração de } \\
\text { informações completas e de } \\
\text { qualidade, e para o compromisso } \\
\text { com o acompanhamento do } \\
\text { paciente e prescrição adequada, } \\
\text { aliada as terapêuticas não } \\
\text { medicamentosas. }\end{array}$ \\
\hline $\begin{array}{l}\text { Takahama } \\
\mathrm{CH} \text { et al. }\end{array}$ & 2014 & $\begin{array}{c}\text { Descrever os dados } \\
\text { epidemiológicos referentes } \\
\text { às exposições a } \\
\text { medicamentos por } \\
\text { mulheres em idade fértil } \\
\text { atendidas por um Centro de } \\
\text { Informações Toxicológicas. }\end{array}$ & $\begin{array}{l}\text { A maioria das ocorrências } \\
(90,5 \%) \text { foi intencional, em } \\
33,7 \% \text { dos casos foram } \\
\text { utilizados de dois a três } \\
\text { medicamentos e a frequência } \\
\text { de hospitalização foi de } \\
\text { 35,6\%. Os medicamentos } \\
\text { com atuação no Sistema } \\
\text { Nervoso Central foram }\end{array}$ & $\begin{array}{l}\text { Para reduzir as intoxicações } \\
\text { agudas em mulheres da faixa } \\
\text { etária suscetível, são necessários } \\
\text { esforços para orientação e } \\
\text { conscientização de profissionais } \\
\text { prescritores e usuários sobre os } \\
\text { riscos do uso indiscriminado } \\
\text { desses fármacos, além de } \\
\text { intervenções para reduzir os } \\
\text { danos por lesões autoinfligidas. }\end{array}$ \\
\hline
\end{tabular}




\begin{tabular}{|c|c|c|c|c|}
\hline & & & $\begin{array}{c}\text { responsáveis por } 59,9 \% \text { das } \\
\text { ocorrências. }\end{array}$ & \\
\hline $\begin{array}{c}\text { Maior } \\
\text { MDCLS }^{21}\end{array}$ & 2015 & $\begin{array}{l}\text { Descrever as internações } \\
\text { hospitalares devidas às } \\
\text { intoxicações } \\
\text { medicamentosas em } \\
\text { menores de cinco anos de } \\
\text { idade no Brasil, de } 2003 \text { a } \\
\text { 2012. }\end{array}$ & $\begin{array}{l}\text { As classes terapêuticas mais } \\
\text { frequentes nas intoxicações } \\
\text { foram fármacos não } \\
\text { especificados ( } 38 \%), \\
\text { antiepilépticos/sedativo- } \\
\text { hipnóticos/antiparkinsoniano } \\
\text { s }(19,8 \%) \text { e antibióticos } \\
\text { sistêmicos (13,4\%), variando } \\
\text { conforme região do país e } \\
\text { idade das crianças. }\end{array}$ & $\begin{array}{l}\text { Restringir o acesso das crianças } \\
\text { aos medicamentos, por meio do } \\
\text { fomento à cultura de segurança } \\
\text { doméstica entre pais e } \\
\text { responsáveis. Também é } \\
\text { necessário discutir a adoção de } \\
\text { embalagens especiais de } \\
\text { proteção à criança nos } \\
\text { medicamentos. }\end{array}$ \\
\hline $\begin{array}{l}\text { Tavares ÉO } \\
\text { et al. }{ }^{22}\end{array}$ & 2013 & $\begin{array}{c}\text { Analisar os fatores } \\
\text { associados à intoxicação } \\
\text { em crianças, a partir de } \\
\text { casos registrados no Centro } \\
\text { de Controle de Intoxicações } \\
\text { do Hospital Universitário } \\
\text { Regional de Maringá }\end{array}$ & $\begin{array}{c}\text { São fatores associados à } \\
\text { intoxicação infantil o sexo } \\
\text { masculino e a faixa etária } 0-4 \\
\text { anos; como fator } \\
\text { predisponente, a residência, } \\
\text { em que a presença do adulto } \\
\text { não impediu a ocorrência; e } \\
\text { desencadeantes o acesso } \\
\text { facilitado a medicamentos e a } \\
\text { exposição oral. }\end{array}$ & $\begin{array}{c}\text { Orientações sobre } \\
\text { acondicionamento de agentes } \\
\text { tóxicos, vigilância das famílias } \\
\text { com conscientização dos riscos } \\
\text { do ambiente doméstico. } \\
\text { Implantação de embalagem de } \\
\text { proteção à criança, com tampa } \\
\text { inviolável e disponibilização de } \\
\text { doses fracionadas. }\end{array}$ \\
\hline $\begin{array}{l}\text { Sales CCF } \\
\text { et } a .^{23}\end{array}$ & 2017 & $\begin{array}{l}\text { Identificar a presença e as } \\
\text { ações de adultos no local da } \\
\text { ocorrência de acidentes } \\
\text { toxicológicos infantis e os } \\
\text { primeiros socorros } \\
\text { realizados. }\end{array}$ & $\begin{array}{l}\text { Maioria dos acidentes } \\
\text { toxicológicos na primeira } \\
\text { infância eram crianças do } \\
\text { sexo masculino, de } 1 \text { a } 2 \\
\text { anos; os principais agentes } \\
\text { foram medicamentos e } \\
\text { predominaram intoxicações } \\
\text { não intencionais. }\end{array}$ & $\begin{array}{l}\text { Oferecimento de capacitação em } \\
\text { primeiros socorros para } \\
\text { acidentes de intoxicação e } \\
\text { envenenamento com crianças } \\
\text { pelos serviços de saúde e abordar } \\
\text { o assunto com a sociedade. }\end{array}$ \\
\hline $\begin{array}{l}\text { Paula et } \\
\text { al. }^{24}\end{array}$ & 2012 & $\begin{array}{c}\text { Determinar as principais } \\
\text { classes terapêuticas } \\
\text { envolvidas nas internações } \\
\text { de idosos decorrentes de } \\
\text { intoxicação e efeito adverso } \\
\text { de medicamentos e seus } \\
\text { agravos. }\end{array}$ & $\begin{array}{c}\text { Há uma tendência crescente } \\
\text { dos problemas associados ao } \\
\text { uso de medicamentos por } \\
\text { idosos e o perfil de consumo } \\
\text { não é suficiente para explicar } \\
\text { as concentrações de casos nas } \\
\text { principais classes } \\
\text { terapêuticas. }\end{array}$ & $\begin{array}{l}\text { Adoção de programas mais } \\
\text { efetivos de farmacovigilância, } \\
\text { capazes de monitorar as } \\
\text { diferentes etapas do processo de } \\
\text { uso de medicamentos: } \\
\text { prescrição, dispensação, } \\
\text { comercialização, administração e } \\
\text { adesão. }\end{array}$ \\
\hline $\begin{array}{c}\text { Toscano } \\
\text { MM et al. }\end{array}$ & 2016 & $\begin{array}{l}\text { Descrever o perfil clínico e } \\
\text { sociodemográfico dos casos } \\
\text { de intoxicações agudas } \\
\text { registradas pelo Ceatox-PB } \\
\text { em } 2012 \text {. }\end{array}$ & $\begin{array}{l}\text { A maioria dos casos de } \\
\text { intoxicação foi relacionada a } \\
\text { medicamentos consumidos } \\
\text { de forma acidental, mais } \\
\text { frequentemente por mulheres } \\
\text { e menores de } 18 \text { anos. }\end{array}$ & $\begin{array}{l}\text { Preenchimento adequado da } \\
\text { ficha de notificação e realização } \\
\text { de medidas preventivas e } \\
\text { profiláticas pelos órgãos } \\
\text { competentes de saúde e } \\
\text { educação. }\end{array}$ \\
\hline $\begin{array}{l}\text { Santos SA } \\
\text { et al. } .^{26}\end{array}$ & 2013 & $\begin{array}{c}\text { Descrever o perfil de } \\
\text { suicídios e tentativas por } \\
\text { intoxicação exógena entre } \\
\text { diferentes sistemas de } \\
\text { informações, para o estado } \\
\text { do Rio de Janeiro. }\end{array}$ & $\begin{array}{l}\text { O sexo feminino, o grupo } \\
\text { etário de } 20-39 \text { anos e o uso } \\
\text { dos agentes tóxicos } \\
\text { predominaram. Mais de } 70 \% \\
\text { dos medicamentos eram } \\
\text { psicotrópicos. O Sinan } \\
\text { apresentou o pior }\end{array}$ & $\begin{array}{c}\text { Resolver os problemas quanto à } \\
\text { cobertura dada pelos sistemas e a } \\
\text { necessidade de aperfeiçoamento } \\
\text { quanto à sua qualidade, de modo } \\
\text { que venham a subsidiar de forma } \\
\text { segura as políticas e ações em } \\
\text { saúde. }\end{array}$ \\
\hline
\end{tabular}




\begin{tabular}{|c|c|c|c|c|}
\hline & & & $\begin{array}{c}\text { desempenho para os agentes } \\
\text { tóxicos. }\end{array}$ & \\
\hline $\begin{array}{l}\text { Mendes LA, } \\
\text { Pereira } \\
\mathrm{BB}^{27}\end{array}$ & 2017 & $\begin{array}{c}\text { Realizar análise } \\
\text { epidemiológica dos casos } \\
\text { de intoxicação por } \\
\text { medicamentos no Brasil } \\
\text { registrados pelo SINITOX. }\end{array}$ & $\begin{array}{c}\text { Maior número de casos na } \\
\text { população feminina, tentativa } \\
\text { de suicídio é a circunstância } \\
\text { mais importante e a } \\
\text { população infantil é a mais } \\
\text { susceptível. }\end{array}$ & $\begin{array}{l}\text { Utilização dos dados do } \\
\text { SINITOX para efetivas } \\
\text { promoções de saúde e adoção de } \\
\text { medidas de vigilância em saúde. }\end{array}$ \\
\hline $\begin{array}{l}\text { Santos SA } \\
\quad \text { et al. } .^{28}\end{array}$ & 2013 & $\begin{array}{l}\text { Estimar a frequência do uso } \\
\text { de substâncias tóxicas } \\
\text { como meio para tentativas e } \\
\text { suicídio, como subsídio } \\
\text { para discutir medidas } \\
\text { preventivas e de restrição. }\end{array}$ & $\begin{array}{l}\text { Cerca de } 70 \% \text { dos indivíduos } \\
\text { que tentaram suicídio } \\
\text { ingeriram substâncias } \\
\text { tóxicas, dessas } 46,2 \% \text { foram } \\
\text { medicamentos, com } \\
\text { predomínio do sexo } \\
\text { masculino e o grupo etário de } \\
30-49 \text { anos. Quanto ao } \\
\text { suicídio, o grupo de mulheres } \\
\text { adolescentes predominou. }\end{array}$ & $\begin{array}{l}\text { Constata-se a necessidade de se } \\
\text { considerar o comportamento } \\
\text { suicida como de fato um } \\
\text { problema de saúde pública em } \\
\text { ascensão no Brasil e investir em } \\
\text { políticas públicas para sua } \\
\text { prevenção. }\end{array}$ \\
\hline
\end{tabular}

Fonte: Dados da pesquisa.

\section{Procedimentos}

A análise estatística e a elaboração dos gráficos e tabelas foram realizadas por meio do software Microsoft Office Excel (2019). Os dados foram analisados quanto às variáveis: ano dos primeiros sintomas, faixa etária, sexo, circunstância, local de exposição, evolução e classificação final. Informações ignoradas ou em branco serão apresentadas.

Foi realizada análise estatística com determinação da média, mediana e desvio padrão dos dados. Para os cálculos de incidência foram utilizados dados demográficos oficiais provenientes do portal Brasil em Síntese, de responsabilidade do Instituto Brasileiro de Geografia e Estatística (IBGE) ${ }^{7}$.

\section{Aspectos Éticos}

Todos os dados utilizados no presente artigo, assim como as publicações da revisão, são provenientes de bases de dados secundárias, de domínio público, que serão apresentadas de forma agregada, impossibilitando a identificação dos sujeitos da pesquisa. Dessa forma, não sendo necessária a apreciação de comitê de ética em pesquisa.

\section{Resultados}

No período analisado, de 2011 a 2017, ocorreram 5.315 intoxicações exógenas por medicamento no município de Brasília, o que corresponde a uma média de 759 casos por ano, a mediana nesses anos foi de 770 casos e o desvio padrão dos dados foi de 275,433 casos. Ademais, a incidência média nessa série temporal foi de 26,6 casos por 100 mil habitantes. Com relação ao sexo, em Brasília, a proporção média de casos foi de 2,07 mulheres (M) intoxicadas para cada homem $(\mathrm{H})$, sendo que essa taxa alcançou seu ápice em 2017, com proporção de 2,54 (M/H). Além disso, ao combinar o sexo com a circunstância da intoxicação medicamentosa, é possível visualizar a frequência na distribuição entre homens e mulheres (Figura 1). Entre os homens, a maioria dos casos é decorrente de intoxicação acidental (36\%), mas com valor semelhante à tentativa de suicídio $(35 \%)$. Contudo, entre as mulheres, os números de tentativa de suicídio são $68,6 \%$ maiores que dos homens, representando $59 \%$ do total de 
intoxicações em mulheres, enquanto a intoxicação acidental representa apenas $18 \%$ dos casos.

Figura 1: Frequência das intoxicações exógenas por medicamentos em Brasília, segundo o sexo e a circunstância do episódio.

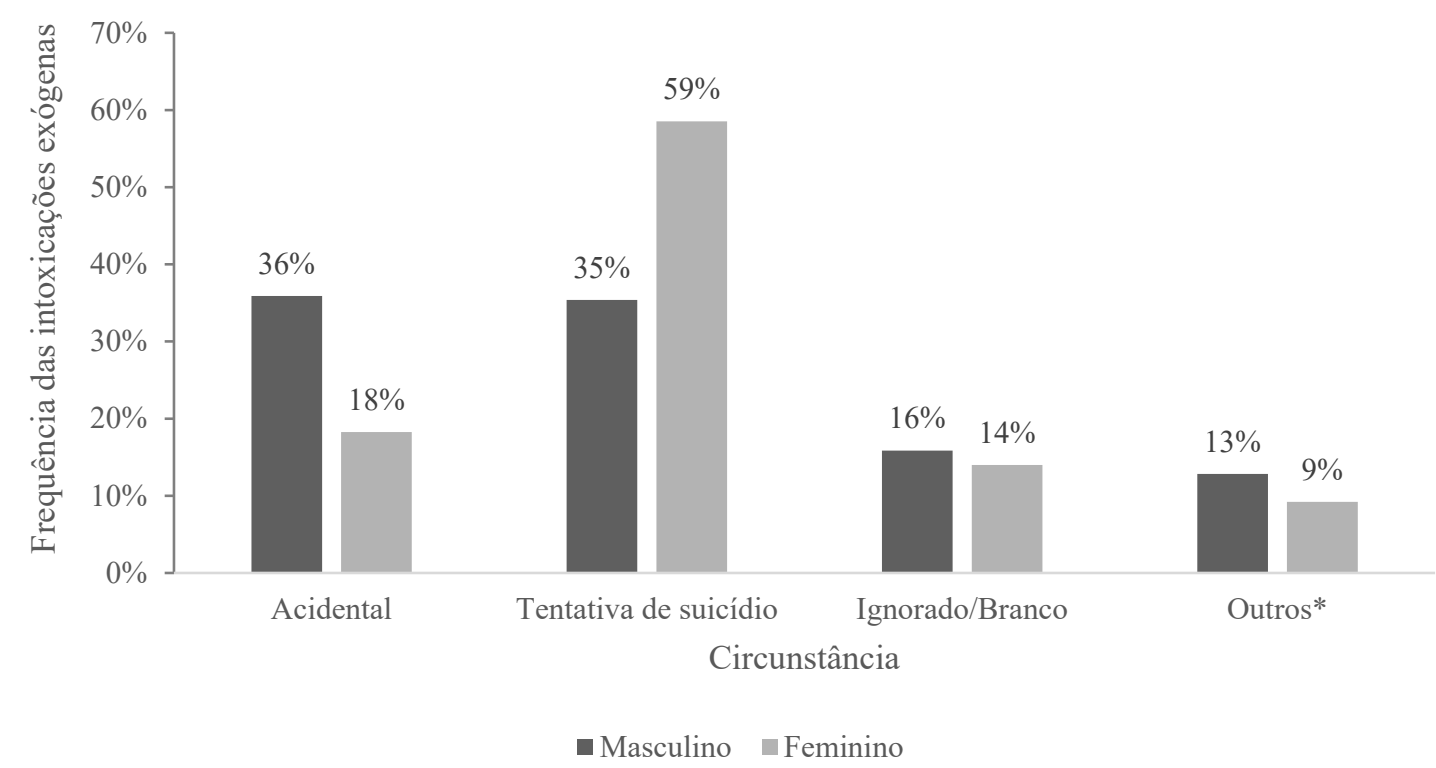

Dados do Sistema de Informação de Agravos de Notificação, Brasília, 2011 a 2017. * Na categoria outros estão inclusos os seguintes grupos: uso habitual, ambiental, uso terapêutico, prescrição médica, erro de administração, automedicação, abuso, ingestão de alimento, tentativa de aborto e violência/homicídio.

Quanto à idade, a faixa etária com mais casos em toda a série histórica analisada foi de 20 a 39 anos e representou $37 \%$ dos casos, sendo que de 2011 a 2013 houve crescimento dos casos notificados nessas idades, seguida de redução até 2015 e novo acréscimo até 2017. A segunda faixa etária com maior prevalência foi de 1 a 4 anos, que representou $20 \%$ dos casos no mesmo período. Contudo, essa faixa etária apresentou crescimento acentuado de 2011 a 2013, quando ultrapassou a faixa etária dos 20 a 39 anos. Felizmente, após esse ano, houve uma redução significativa dos casos até 2015, e, em 2017, a faixa etária de 1 a 4 anos ocupa a quarta posição entre as demais (Figura 2).

Figura 2: Número total de casos de intoxicações exógenas por medicamentos em Brasília, segundo a faixa etária e ano da ocorrência do episódio.

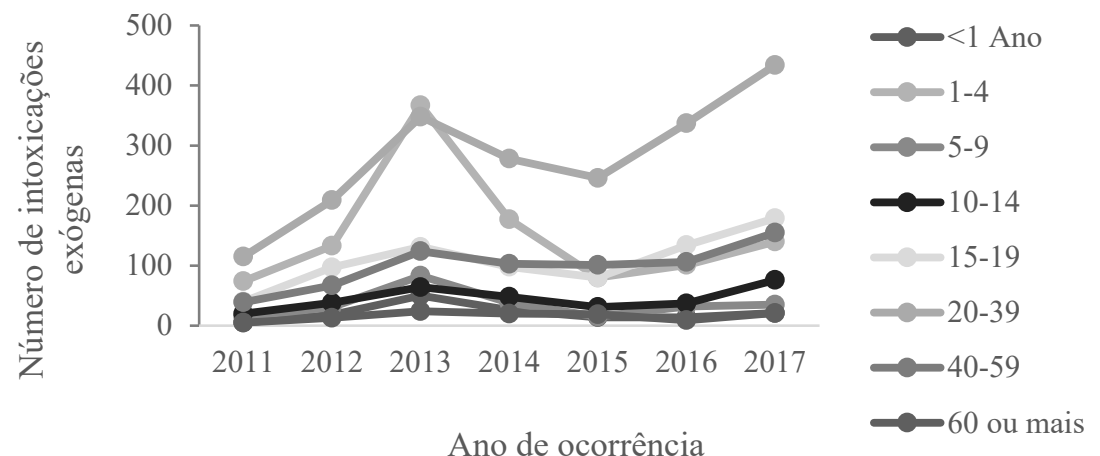

Dados do Sistema de Informação de Agravos de Notificação, Brasília, 2011 a 2017. 
A maioria das intoxicações medicamentosas ocorridas de 2011 a 2017 foi aguda, única e representou $57 \%$ do total (Tabela 1). Contudo, houve uma quantidade significativa de casos nos quais essa categoria foi ignorada ou deixada em branco, o que prejudica uma análise mais abrangente do tipo de exposição. Além disso, apesar dos casos de exposição aguda repetida representarem apenas $7 \%$ dos casos, $87 \%$ deles são decorrentes de tentativa de suicídio.

Tabela 1: Frequência das intoxicações exógenas por medicamento, segundo o tipo da exposição. Dados do Sistema de Informação de Agravos de Notificação, Brasília, 2011 a 2017.

\begin{tabular}{ccccccccc}
\hline Tipo de exposição & $\mathbf{2 0 1 1}$ & $\mathbf{2 0 1 2}$ & $\mathbf{2 0 1 3}$ & $\mathbf{2 0 1 4}$ & $\mathbf{2 0 1 5}$ & $\mathbf{2 0 1 6}$ & $\mathbf{2 0 1 7}$ & Total \\
\hline Aguda-única & $42 \%$ & $43 \%$ & $71 \%$ & $77 \%$ & $47 \%$ & $51 \%$ & $50 \%$ & $57 \%$ \\
\hline Aguda-repetida & $2 \%$ & $5 \%$ & $5 \%$ & $9 \%$ & $7 \%$ & $7 \%$ & $12 \%$ & $7 \%$ \\
\hline Crônica & $0 \%$ & $0 \%$ & $0 \%$ & $1 \%$ & $1 \%$ & $1 \%$ & $1 \%$ & $1 \%$ \\
\hline Aguda sobre crônica & $0 \%$ & $0 \%$ & $1 \%$ & $1 \%$ & $1 \%$ & $0 \%$ & $0 \%$ & $0 \%$ \\
\hline Ignorado ou em branco & $56 \%$ & $52 \%$ & $23 \%$ & $12 \%$ & $44 \%$ & $41 \%$ & $36 \%$ & $34 \%$ \\
\hline
\end{tabular}

Dentre todas as intoxicações notificadas de 2011 a 2017, 72\% delas foram confirmadas, $11 \%$ delas foram apenas exposição ao agente tóxico, no entanto, essa informação foi omitida em $15 \%$ dos casos. Tendo em vista esses aspectos, o principal critério de confirmação em todos os anos foi o clínico, em $68 \%$ deles, enquanto os demais foram pouco utilizados. Mas, em 2012 e 2013, o critério clínico-epidemiológico também foi relevante nas confirmações e representou $27,9 \%$ e $23,9 \%$ dos casos em cada ano, respectivamente (Figura 3).

Figura 3: Critério de confirmação de casos de intoxicações exógenas por medicamentos em Brasília segundo o ano da ocorrência.

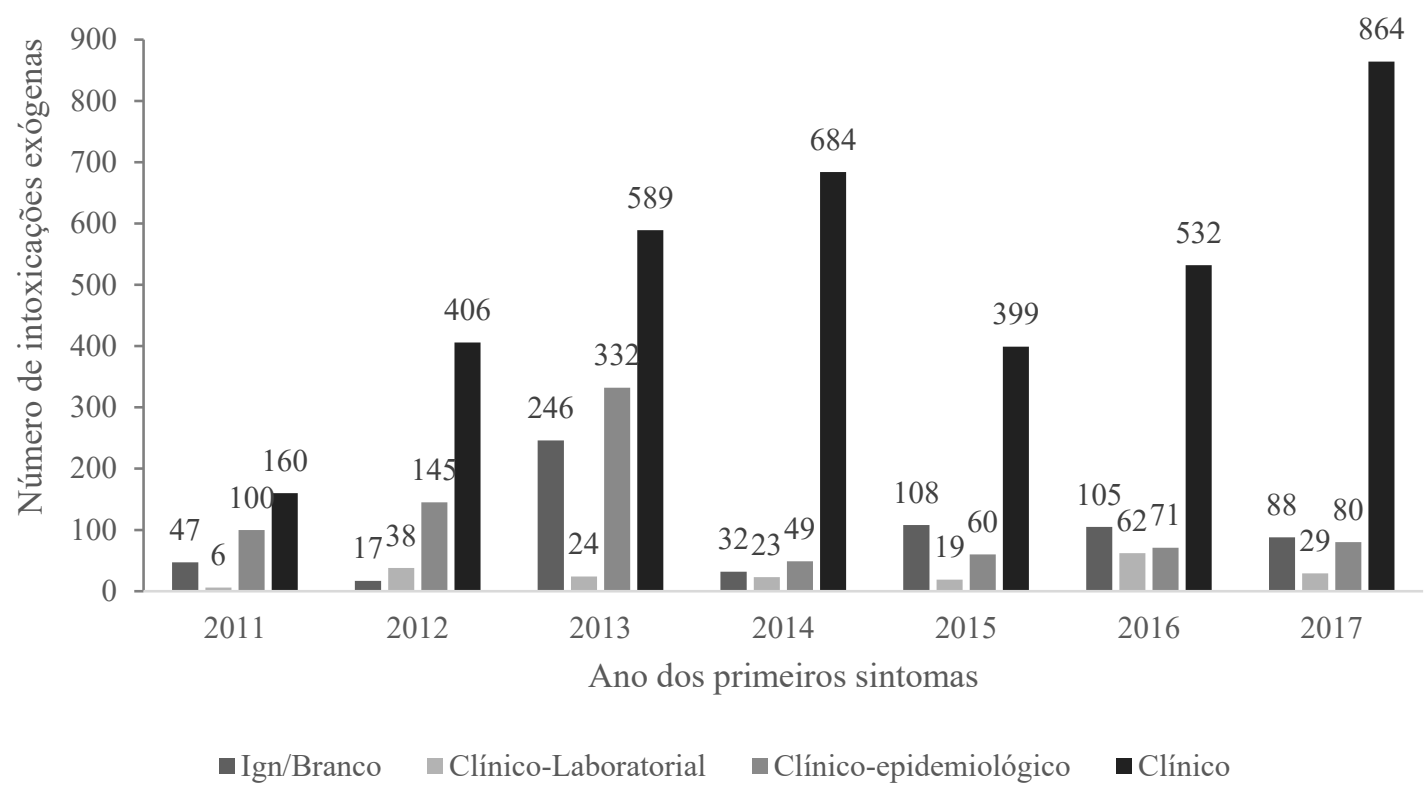

Dados do Sistema de Informação de Agravos de Notificação, Brasília, 2011 a 2017. Ign/ = Ignorado. 
Após a intoxicação por medicamento, a evolução dos casos não foi notificada em $40 \%$ deles. Nesse sentido, os dados demonstram que $57 \%$ dos casos evoluíram para cura, e uma quantidade menor que $1 \%$ evoluiu com sequela ou óbito. Ao considerar o ano de 2014, que teve a menor taxa de ignorados, de $11 \%$, o número de casos que evoluíram com cura sem sequelas foi de $85 \%$, cura com sequela $1 \%$ e óbitos devido à intoxicação ou por outra causa $0,5 \%$ cada. Logo, é possível observar que a maioria dos casos de intoxicação por medicamento, em Brasília, evoluem de forma favorável (Tabela 2).

Tabela 2: Frequência das intoxicações exógenas por medicamento conforme evolução. Dados do Sistema de Informação de Agravos de Notificação, Brasília, 2011 a 2017.

\begin{tabular}{cllllllll}
\hline Evolução & $\mathbf{2 0 1 1}$ & $\mathbf{2 0 1 2}$ & $\mathbf{2 0 1 3}$ & $\mathbf{2 0 1 4}$ & $\mathbf{2 0 1 5}$ & $\mathbf{2 0 1 6}$ & $\mathbf{2 0 1 7}$ & Total \\
\hline Cura sem sequela & $45 \%$ & $35 \%$ & $66 \%$ & $85 \%$ & $52 \%$ & $49 \%$ & $51 \%$ & $57 \%$ \\
\hline Cura com sequela & $0 \%$ & $0 \%$ & $1 \%$ & $1 \%$ & $1 \%$ & $0 \%$ & $1 \%$ & $1 \%$ \\
\hline Óbito por intoxicação exógena & $1 \%$ & $0 \%$ & $0 \%$ & $1 \%$ & $0 \%$ & $0 \%$ & $0 \%$ & $0 \%$ \\
\hline Óbito por outra causa & $0 \%$ & $0 \%$ & $0 \%$ & $1 \%$ & $0 \%$ & $0 \%$ & $0 \%$ & $0 \%$ \\
\hline Perda de Seguimento & $0 \%$ & $0 \%$ & $2 \%$ & $2 \%$ & $3 \%$ & $1 \%$ & $2 \%$ & $1 \%$ \\
\hline Ignorado ou em branco & $54 \%$ & $65 \%$ & $31 \%$ & $11 \%$ & $44 \%$ & $49 \%$ & $46 \%$ & $40 \%$ \\
\hline
\end{tabular}

Por fim, ao analisar se a exposição foi no trabalho, novamente a quantidade de dados ignorados ou em branco é de $31,58 \%$, considerando toda a série histórica analisada e nenhum caso reportado foi relacionado ao trabalho.

\section{Revisão Bibliográfica}

A revisão bibliográfica de literatura permitiu identificar um total de 158 publicações nas plataformas Scielo e LILACS a partir dos descritores "intoxicação" e "medicamentos". Durante a fase de seleção, 102 resultados foram excluídos por serem anteriores a 2012 ou de idiomas diferentes de português e inglês. Também foram excluídas 23 duplicatas. Por fim, 11 artigos foram excluídos por não abordarem intoxicações exógenas ou não trazerem propostas de intervenção (Figura 4).

Figura 4: Fluxograma da revisão de literatura. Fonte: Própria.

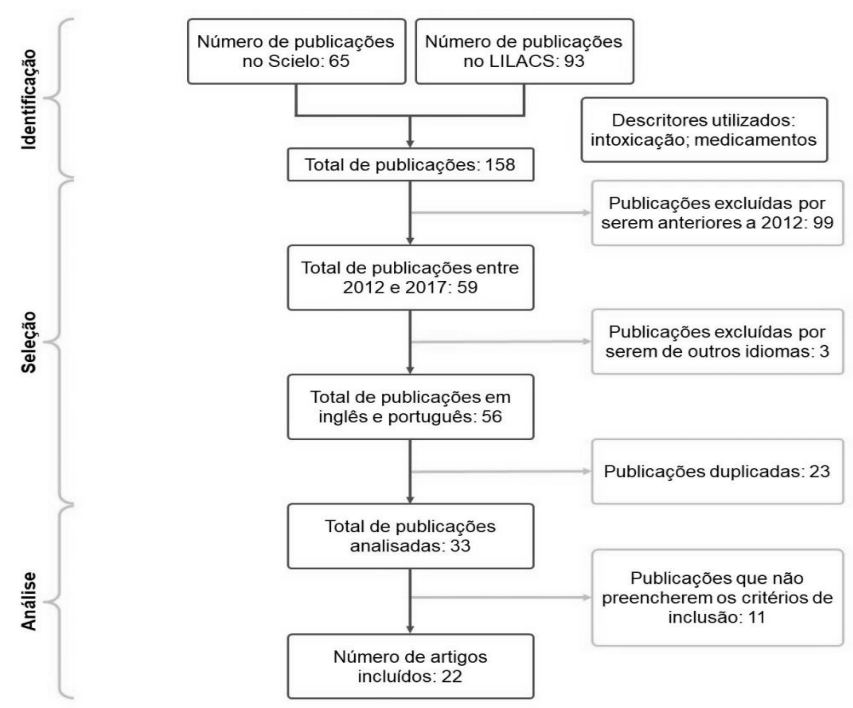


Logo, ao final, 22 publicações atenderam aos critérios de análise e foram agrupadas em um quadro com as seguintes informações: título, autores, ano de publicação, objetivo geral, principais resultados e proposta de intervenção (Quadro 1). Essas publicações serviram como base para elaboração da discussão e da proposta de intervenção do presente artigo e abrangem o tema das intoxicações exógenas por medicamento em diferentes populações, principalmente na pediátrica; com desenhos em sua maioria do tipo observacional; e algumas com enfoque em suicídio.

\section{Discussão}

Segundo Amorim et al. ${ }^{8}$, as intoxicações exógenas são problemas de saúde pública extremamente relevantes no Brasil, uma vez que apresentam elevada frequência e morbidade na população geral do país. Conforme a base do Sistema Nacional de Informações Tóxico Farmacológicas do ano de 2017, observouse que mais de $27 \%$ das intoxicações registradas no país foram por medicamentos e $25 \%$ dos óbitos causados por intoxicação humana foram decorrentes desse mesmo agente $^{2}$.

O Distrito Federal é uma unidade federativa com considerável número de casos de intoxicação exógena durante um período significativo. Com o intuito de reduzir esses números foi implementado o Disque-Intoxicação, um 0800 coordenado pela Rede Nacional de Centros de Informação e Assistência Toxicológica (Renaciat) que visa prestar esclarecimentos à população $\mathrm{e}$ facilitar $\mathrm{o}$ fornecimento dos primeiros socorros e a prescrição do tratamento terapêutico oportuno para cada categoria de substância tóxica. Contudo, apesar do número ser obrigatoriamente advertido em rótulos e bulas dos produtos regulamentados pela Anvisa e em informes indicativos em clínicas, hospitais e laboratórios, o Disque-Intoxicação ainda não é amplamente conhecido pela população ${ }^{9}$.

Brasília, quando comparada com outras capitais de mesmo porte, no mesmo período, apresenta prevalência média anual de intoxicações por medicamentos menor que a de Belo Horizonte, que apresentou 27,2 casos por cem mil habitantes, e maior que Salvador e Fortaleza com 12,9 e 9,1 casos por cem mil habitantes, respectivamente. No entanto, em relação às unidades federativas, o Distrito Federal é o $10^{\circ}$ com maior incidência de intoxicações por medicamentos, uma posição razoavelmente expressiva, na primeira colocação está o Espírito Santo com 74,1 casos por cem mil habitantes e por último o Amapá, com taxa de $0,6^{4,6}$.

$\mathrm{O}$ alto número de casos relacionados a essa categoria de agentes está relacionado a tentativas de suicídio, nas quais as intoxicações por medicamentos e outras substâncias tóxicas são os métodos de autoextermínio utilizados predominantemente por mulheres e geralmente na faixa etária dos 20 a 59 anos; $\mathrm{e}$ à exposição acidental, geralmente vinculada a crianças menores que cinco anos e idosos 5,10 .

O presente estudo evidenciou que os casos de intoxicação exógena por medicamentos, de maneira geral, foram predominantes no sexo feminino, com maior porcentagem de casos cuja circunstância do envenenamento era por tentativa de suicídio, com significativa diferença para intoxicações acidentais. Com relação ao sexo masculino, é possível concluir que ambas as eventualidades são igualmente relevantes para o contexto de envenenamento exógeno.

Os achados de um estudo realizado por Magalhães et al. ${ }^{1}$ em Arapiraca, localizada no estado de Alagoas, a $136 \mathrm{~km}$ de Maceió, que abordavam os atendimentos a tentativas de suicídio mostraram que as mulheres adultas tendem a cometer mais 
tentativas de suicídio do que os homens e usualmente utilizam a intoxicação por medicamentos, por se tratar de um meio menos invasivo, o qual não afetaria a estética. Uma análise desenvolvida por Vieira et al. ${ }^{11}$, no município de Barra do Garças, no estado do Mato Grosso, também evidenciou a prevalência no sexo feminino. Isso reflete uma tendência, percebida na literatura e constatada por essa análise, das mulheres serem mais propensas a realizar tentativas de suicídio por meio do autoenvenenamento.

No entanto, a maioria dos artigos revisados não mostrou semelhança entre a porcentagem de casos em que a causa da intoxicação foi tentativa de suicídio e de eventos referentes a causas acidentais observadas no presente estudo. Contudo, um artigo elaborado por Mota et al. ${ }^{5}$ expressa que a taxa de mortalidade no Brasil por suicídio foi superior, em ambos os sexos, as outras circunstâncias de óbito por envenenamento exógeno. Ademais, na categoria das intoxicações acidentais, as taxas de mortalidade para os homens foram maiores em relação às das mulheres, o que poderia explicar a equivalência numérica entre a porcentagem de casos por tentativas de autoextermínio e por causas acidentais, o que significa que ambas as causas são relevantes.

Já em relação à faixa etária em que tais eventos tóxicos ocorrem, essa análise mostrou que o número de casos por intoxicação exógena notificados foi predominante na faixa etária de 20 a 39 anos de idade, seguida pelo intervalo etário de 1 a 4 anos. Conforme Mota et al. ${ }^{5}$, a primeira está correlacionada à exposição suicida/intencional, geralmente correspondendo a maioria das pessoas que morrem por intoxicação exógena.

A segunda é caracteristicamente associada a envenenamento exógeno acidental, ocasionado principalmente por fatores intrínsecos ao desenvolvimento das crianças, como a exploração do ambiente e a curiosidade característica dessa faixa etária, que eventualmente pode levar os integrantes dessa faixa etária a ingerir substâncias e objetos, de acordo com Magalhães et al. ${ }^{1}$. Além disso, Domingos et $a{ }^{12}$ destacam que existem fatores relacionados aos medicamentos que contribuem para a relevância da intoxicação exógena em crianças, os quais são a falta de controle na comercialização dos medicamentos, presença de fármacos prescritos para adultos no ambiente doméstico, geralmente ao alcance da criança, o uso dessas drogas na frente da criança, a própria oferta de medicamentos prescritos para as crianças com o argumento que são balas ou doces, supervisão inadequada dos integrantes pertencentes a essa faixa etária e o descuido na guarda e descarte desses produtos.

Ademais, neste estudo também é possível perceber que as categorias presentes na ficha de notificação obtidas por meio do SINAN, como a informação sobre o tipo de exposição, o critério de confirmação de intoxicação exógena, a evolução do caso e se a exposição foi no trabalho, apresentaram significativa quantidade de informações ignoradas ou em branco. Isso reflete a subnotificação causada pela falta de preenchimento completo da ficha de notificação compulsória, o que retrata uma adversidade de sensibilização dos profissionais de saúde com relação à importância do preenchimento correto da ficha de notificação ${ }^{10}$.

$$
\text { Portanto, as variáveis }
$$
intrinsecamente referentes ao contexto de intoxicação exógena por medicamentos são principalmente a idade e o sexo do paciente, as quais predispõem certos tipos de circunstâncias, essencialmente a acidental e a intencional/suicida. Além disso, o preenchimento adequado da ficha causa grande impacto na coleta e utilização de dados referentes ao contexto de envenenamento exógeno, o que exige que as medidas de intervenção sejam vinculadas a esses aspectos.

Por conseguinte, as intervenções que poderiam ser aplicadas com o intuito de 
melhorar a realidade das interações medicamentosas em Brasília devem ser embasadas em literatura científica, as quais mostrem eficácia nos respectivos estudos. No entanto, durante a revisão sistematizada, os artigos encontrados não abordavam a implementação e resultados das medidas apresentadas para redução do número de casos, o que configura uma limitação deste estudo.

Logo, é necessário desenvolver estratégias de promoção de qualidade de vida, de educação, de proteção e de recuperação da saúde e de prevenção de danos $^{13-15}$, promover a educação permanente dos profissionais de saúde das unidades de atenção básica, inclusive do Programa Saúde da Família, dos serviços de saúde mental, das unidades de urgência e emergência, de acordo com os princípios da integralidade e da humanização ${ }^{8,} 12,13,16$. Além disso, fortalecer a educação em saúde com campanhas educativas ${ }^{8,}$ 16-20, bem como a fiscalização por parte dos órgãos governamentais ${ }^{16}$, visando à melhoria da qualidade de vida da população podem contribuir com a redução do número de casos por intoxicação exógena.

Com relação à abordagem específica do suicídio é fundamental aprimorar estratégias de informação, de comunicação e de sensibilização da sociedade de que o autoextermínio é um problema de saúde pública prevenível ${ }^{16}$. Outras medidas que poderiam ser adotadas são a capacitação dos profissionais de saúde no manejo do comportamento suicida ${ }^{1}$; a utilização de práticos instrumentos validados para detecção de ideação suicida durante a anamnese, principalmente em pacientes com fatores de risco para $\mathrm{tal}^{16}$; a organização de uma linha de cuidados integrais (promoção, prevenção, tratamento e recuperação) em todos os níveis de atenção ${ }^{14}$, garantindo o acesso às diferentes modalidades terapêuticas, com intuito de melhorar assim o sistema de saúde, para que possa garantir o acesso precoce a avaliações clínicas adequadas, o que assegura suporte psicológico e/ou psiquiátrico ao paciente com comportamento suicida ${ }^{11,14,15}$; e o aumento da segurança e da efetividade dos tratamentos para os transtornos psiquiátricos com alto risco de suicídio.

Ademais, outras providências podem contribuir para redução das taxas de tentativa de suicídio como a identificação da prevalência dos determinantes e condicionantes do suicídio e tentativas, assim como os fatores protetores ${ }^{14}, 16$; o desenvolvimento de ações intersetoriais de prevenção ao suicídio $^{16}$, de responsabilidade pública, sem excluir a responsabilidade de toda a sociedade; e a realização de projetos educativos, com o intuito de aumentar o conhecimento público e profissional dos fatores de risco para o autoextermínio $^{1,16}$. O desenvolvimento de investigações sobre a prevenção de suicídio $^{14}$, as quais através de pesquisas médicas podem esclarecer os padrões locais, regionais e nacionais de suicídio, os mecanismos desencadeadores ${ }^{16}$, benefícios e riscos específicos dos tratamentos médicos e intervenções psicossociais ${ }^{14,} 16$ que possam prevenir o autoextermínio, contribuiria também para redução do número de suicídios.

Em relação às medidas voltadas para prevenção de circunstâncias acidentais, principalmente relativas à faixa etária infantil, são implantação de embalagens de medicamentos com objetivo de proteger a criança, com tampa inviolável e oferta de doses fracionadas ${ }^{8,12,21,22}$, com o intuito de dificultar o acesso dessas crianças a esses agentes tóxicos. O papel do Estado é essencial através do apoio legislativo, bem como $\mathrm{o}$ da indústria que produz as embalagens desses agentes ${ }^{8,12}$. Além disso, instruções para os pais sobre acondicionamento adequado de agentes tóxicos e vigilância das famílias com conscientização dos riscos do ambiente doméstico $^{22}$ são fundamentais para prevenção desse tipo de intoxicações. Capacitações em primeiros socorros necessários para manejo desses agravos e melhoria do atendimento emergencial também são medidas bastante eficazes para 
redução da mortalidade por intoxicação medicamentosa ${ }^{12,23}$.

Outras providências que potencialmente contribuiriam para redução dos casos de intoxicação exógena seriam o fortalecimento de campanhas de saúde de conscientização voltadas para pacientes, prescritores e dispensadores de fármacos ${ }^{16}$, 20, 24; a consolidação de programas de farmacovigilância, os quais monitorariam todo o processo de uso de medicamentos, compreendendo assim a prescrição, dispensação, comercialização, administração e adesão ao tratamento proposto; ${ }^{16,24}$ e a mudança os padrões de consumo de medicamentos por alterações legislativas, com consequente maior fiscalização por parte dos órgãos governamentais $^{11,16}$.

Ademais, é primordial que ocorra investimento da Vigilância em Saúde na sensibilização continuada dos profissionais da Atenção Básica para melhorar a notificação desse agravo ${ }^{1,10,19,21,25,26}$. A ampliação da integração entre os diversos sistemas de informação de vigilância desses agravos $^{5,} 8,27$ também é uma medida necessária e proporcionaria análise completa e adequada das informações colhidas, o que facilita o planejamento e desenvolvimento de ações voltadas para a prevenção dos riscos e consequências dessas intoxicações medicamentosas.

\section{Conclusão}

O estudo mostrou que no período de 2011 a 2017 ocorreram 5.315 intoxicações exógenas por medicamento no município de Brasília, sendo que a prevalência média nessa série temporal foi de 26,6 casos por cem mil habitantes. Nota-se que o alto número de casos está relacionado a tentativas de suicídios, nas quais as intoxicações por medicamentos e outras substâncias tóxicas são os métodos de autoextermínio utilizados e à exposição acidental, geralmente vinculada a crianças menores que cinco anos e idosos.

Além disso, é interessante ressaltar como uma limitação a existência de uma possível subnotificação, nos anos anteriores a 2011, dos casos de intoxicação no sistema TabNet. Ademais, durante a revisão sistematizada os artigos encontrados não abordavam a implementação e resultados das medidas apresentadas para redução do número de casos, o que também configura uma limitação deste estudo.

Diante do estudo, é possível observar que medidas de intervenção visam mitigar esses índices, como o DisqueIntoxicação, todavia ainda não é amplamente conhecido pela população. Ademais, observa-se a importância de criar e implementar medidas voltadas para prevenção de circunstâncias acidentais, principalmente relativas à faixa etária infantil e desenvolver estratégias de promoção de qualidade de vida, de educação, de proteção e recuperação da saúde e de prevenção de danos relacionados ao suicídio e demais consequências da intoxicação exógena por medicamentos.

\section{Referências}

1. Magalhães APNd, Alves VdM, Comassetto I, Lima PC, Faro ACMe, Nardi AE. Atendimento a tentativas de suicídio por serviço de atenção pré-hospitalar. $\mathrm{J}$ bras psiquiatr. 2014;63(1):16-22.

2. Fundação Oswaldo Cruz/Centro de Informação Científica e Tecnológica/Sistema Nacional de Informações Tóxico-Farmacológicas. Casos, Óbitos e Letalidade de Intoxicação Humana por Agente e por Região. Brasil, 2017. Rio de Janeiro: MS/FIOCRUZ/SINITOX; 2020. 
3. Klinger EI, Schmidt DC, Lemos DB, Pasa L, Possuelo LG, Valim ARM. Intoxicação exógena por medicamentos na população jovem do Rio Grande do Sul. Revista de Epidemiologia e Controle de Infecção. 2016;6 (suplemento): 42-52.

4. Brasil. Ministério da Saúde. Secretaria de Vigilância em Saúde. Sistema de Informação de Agravos de Notificação - Sinan: normas e rotinas. 2 ed. Brasília: Editora do Ministério da Saúde; 2007.

5. Mota DM, Melo JRR, Freitas DRCd, Machado M. Perfil da mortalidade por intoxicação com medicamentos no Brasil, 1996-2005: retrato de uma década. Ciênc saúde coletiva. 2012;17(1):61-70.

6. Ministério da Saúde. Indicadores e Dados básicos - IDB/SUS. Brasília; 2012 [15 jun. 2019]. Available from: http://tabnet.datasus.gov.br/cgi/idb2012/matriz.htm\#demog.

7. Instituto Brasileiro de Geografia e Estatística. O Brasil em síntese. Brasília; 2017 [15 jun. 2019]. Disponível em: https://cidades.ibge.gov.br/brasil/df/brasilia/panorama.

8. Amorim MLP, Mello MJGd, Siqueira MTd. Poisoning in children and adolescents notified at a toxicology center in the Northeast of Brazil. Rev Bras Saude Mater Infant. 2017;17(4):765-72.

9. Brasil. Portal Agência Nacional de Vigilância Sanitária [online]. Disque-intoxicação: 2020. [17 jun. 2019]. Available from: http://portal.anvisa.gov.br/disqueintoxicacao.

10. Teles AdS, Oliveira RFdA, Coelho TCB, Ribeiro GV, Mendes WML, Santos PNP. Papel dos medicamentos nas intoxicações causadas por agentes químicos em município da Bahia, no período de 2007 a 2010. Rev ciênc farm básica apl. 2013;34(2).

11. Vieira LP, Santana VTPd, Suchara EA. Caracterização de tentativas de suicídios por substâncias exógenas. Cad saúde colet. 2015;23(2):118-23.

12. Domingos SM, Borghesan NBA, Merino MdFGL, Higarashi IH. Internações por intoxicação de crianças de zero a 14 anos em hospital de ensino no Sul do Brasil, 20062011. Epidemiologia e Serviços de Saúde. 2016;25(2):343-50.

13. Antunes F, Félix de Oliveira ML. Characteristics of patients hospitalized at an intensive care unit due to drug abuse. Investigación y Educación en Enfermería. 2013;31(2):2019.

14. Carvalho ILdN, Lôbo APA, Aguiar CAdA, Campos AR. Suicidally motivated intoxication by psychoactive drugs: characterization among the elderly. Rev bras geriatr gerontol. 2017;20(1):129-37.

15. Moreira DL, Cavalcante Martins M, do Amaral Gubert F, Pereira de Sousa FS. Perfil de pacientes atendidos por tentativa de suicídio em um centro de assistência toxicológica. Cienc enferm. 2015;21(2):63-75.

16. Oliveira JdFMd. Internações hospitalares e mortalidade por intoxicação medicamentosa em São Paulo. 2017. p. 128-.

17. Carvalho IV. Incidência e caracterização de eventos adversos aos medicamentos (EAM) na unidade de emergência referenciada pediátrica do Hospital de Clínicas da UNICAMP. 2013. p. 95-.

18. Oliveira FFS, Suchara EA. Epidemiological profile of exogenous poisoning in children and adolescents from a municipality in the state of Mato Grosso. Rev paul pediatr. 2014;32(4):299-305.

19. Oliveira JdFMd, Wagner GA, Romano-Lieber NS, Antunes JLF. Tendência da mortalidade por intoxicação medicamentosa entre gêneros e faixas etárias no Estado de São Paulo, Brasil, 1996-2012. Ciência \&amp; Saúde Coletiva. 2017;22(10):3381-91.

20. Takahama CH, Turini CA, Girotto E. Perfil das exposições a medicamentos por mulheres em idade reprodutiva atendidas por um Centro de Informações Toxicológicas. Ciência \&amp; Saúde Coletiva. 2014;19(4):1191-9. 
21. Maior MdCLS. Internações hospitalares de crianças menores de cinco anos por intoxicações medicamentosas no Brasil. 2015. p. 168-.

22. Tavares ÉO, Buriola AA, Santos JAT, Ballani TdSL, Oliveira MLFd. Fatores associados à intoxicação infantil. Esc Anna Nery Rev Enferm. 2013;17(1):31-7.

23. Sales CCF, Suguyama P, Guedes MRJ, Borghesan NBA, Higarashi IH, Oliveira MLFd. Intoxicação na primeira infância: socorros domiciliares realizados por adultos. Rev baiana enferm. 2017;31(4):e23766-e.

24. Paula TCd, Bochner R, Montilla DER. Análise clínica e epidemiológica das internações hospitalares de idosos decorrentes de intoxicações e efeitos adversos de medicamentos, Brasil, de 2004 a 2008. Rev bras epidemiol. 2012;15(4):828-44.

25. Toscano MM, Landim JTA, Rocha AB, Sousa-Muñoz RLd. Intoxicações exógenas agudas registradas em Centro de Assistência Toxicológica. Saude e pesqui (Impr). 2016;9(3):425-32.

26. Santos SA, Legay LF, Lovisi GM, Santos JFdC, Lima LA. Suicídios e tentativas de suicídios por intoxicação exógena no Rio de Janeiro: análise dos dados dos sistemas oficiais de informação em saúde, 2006-2008*. Rev bras epidemiol. 2013;16(2):376-87.

27. Mendes LA, Pereira BB. Intoxicações por medicamentos no Brasil registradas pelo SINITOX entre 2007 e 2011. J Health Biol Sci (Online). 2017;5(2):165-70.

28. Santos SA, Legay LF, Lovisi GM. Substâncias tóxicas e tentativas e suicídios: considerações sobre acesso e medidas restritivas. Cad saúde colet. 2013;21(1):53-61.

Como citar este artigo:

Soares JYS, Lima BM, Verri IA, Stefan Vilges de Oliveira SV. Perfil epidemiológico de intoxicação exógena por medicamentos em Brasília. . Rev. Aten. Saúde. 2021; 19(67): 202217. 